\title{
Preventing Deaths of Adolescents through Targeted Investments: World Health Organization
}

\section{Saurabh RamBihariLal Shrivastava* and Prateek Saurabh Shrivastava}

Department of Community Medicine, Shri Sathya Sai Medical College and Research Institute, Tamil Nadu, India

Globally, it is not a hidden secret that the special needs of adolescents have either been ignored or not understood by the policy makers [1]. This is clearly evident by the recent facts which suggest that in excess of 3000 adolescents die on a daily basis, predominantly from preventable causes [2]. In-fact, almost $67 \%$ of the reported deaths are in low and middle income nations, with road traffic injury and lower respiratory infections being the commonest cause of deaths among male and female adolescents respectively worldwide [2]. At the same time, it is important to realize that most of the adolescents are unable to avail the desired prevention and care services, either due to lack of awareness or due to the non-existence of the same [1-3].

However, considering the reality that most of the reported deaths could be averted with the help of quality assured health care, education and social support, a definite change is seen with adolescent health being incorporated as an essential element in the global strategy for the welfare of women, children and adolescents [13]. In addition, there is a definite necessity to invest in resources for ensuring betterment in the adolescent health standards as they are a vulnerable population group with special needs, have a fundamental right to health, can result in triple health benefits (viz. now, in their future and for the next generation), minimize present and future health expenditures, and accounts for a significant proportion of global morbidity and mortality [2].

As adolescents' health and well-being remain the key factors for a healthier and more sustainable society, the World Health Organization has released a set of guidelines to provide directives to different stakeholders [4]. It has been emphasized that betterment in the quality of the health system has been just one component, nevertheless for the holistic improvement there is a need to involve parents, families and the community as well $[1,4]$. These formulated guidelines provide comprehensive information to the policy makers to decide what to do for adolescent health and how to go about it [4]. There is a definitive emphasis on the fact that adolescents are the key asset of the society and that their contribution in the society can be enhanced through their holistic participation [2].

The need of the hour is to ensure that national policy frameworks acknowledge the significance of the meaningful engagement of adolescents, involve adolescents in policy making meant for their welfare and even in their implementation at different levels, train youth leaders to develop their competencies and play an effective role in improvement of their health and wellbeing, improve literacy status and legal knowledge among adolescents about their rights, formulate mechanisms to encourage their participation in health services, and develop monitoring and evaluation strategies for their involvement [1-4]. Further, interventions should target all the concerned sectors ranging from comprehensive sexuality education in schools, raising the age bar for alcohol consumption, compulsory use of seat-belts or helmets, etc. $[2,4]$.

To conclude, even small investments on adolescents will play a crucial role in the development of a healthy and empowered adult who can significantly contribute towards the welfare of their society, coming generations and the nation.

\section{References}

1. Shrivastava SR, Shrivastava PS, Ramasamy J (2016) Why not universally empower adolescents? Commitment by the World Health Organization. Int $J$ Adv Med Health Res 3: 46-47.

2. World Health Organization (2017) More than 1.2 million adolescents die every year, nearly all preventable.

3. Shrivastava SR, Shrivastava PS, Ramasamy J (2017) Improving adolescent health by meeting global standards set by the World Health Organization. Ann Trop Med Public Health 10: 284-285.

4. World Health Organization (2017) Global accelerated action for the health of adolescents (AA-HA!) - Guidance to support country implementation.
*Corresponding author: Saurabh RamBihariLal Shrivastava, Department of Community Medicine, Shri Sathya Sai Medical College and Research Institute, Tamil Nadu, India, Tel: 919884227224; Email: drshrishri2008@gmail.com

Received: July 30, 2018; Accepted: August 06, 2018; Published: August 13, 2018

Citation: Shrivastava SR, Shrivastava PS (2018) Preventing Deaths of Adolescents through Targeted Investments: World Health Organization. Prim Health Care 8: 124. doi: $10.4172 / 2167-1079.1000 \mathrm{e} 124$

Copyright: () 2018 Shrivastava SR, et al. This is an open-access article distributed under the terms of the Creative Commons Attribution License, which permits unrestricted use, distribution, and reproduction in any medium, provided the original author and source are credited. 